Janine Jagger, MPH; Richard D. Pearson, MD

\title{
A View From the Cutting Edge
}

It is clear that there is a rising tide of concern about the long-standing problem of needlestick injuries sustained by health care workers. Perhaps even more remarkable than the current level of concern was the relative lack of it prior to the advent of AIDS. Needled devices have posed a significant risk of disease transmission for as long as they have been used in health care settings. The possible, yet improbable, transmission of HIV has not substantially altered the risk of disease transmission by needlestick. Nevertheless, it has captured the attention of the health care community and considerably lowered our tolerance for the hazards of contaminated needles. In that respect, the current climate may represent an historic opportunity to make significant advances in creating a safer environment for health care workers.

The articles by Ribner and colleagues, and by Krasinski and colleagues in this issue are excellent examples of the quantitative assessment of new needlestick prevention strategies and as such, represent valuable contributions to the growing body of literature devoted to this problem. The striking similarities in the purpose and findings of these studies are instructive as to what can be expected when disposable cardboard containers are replaced by rigid, puncture resistant, disposal containers. The more subtle differences in the conclusions of the two studies are also revealing, demonstrating the consequences of different methods of gathering and reporting data.

The two studies are complementary in their basic findings. The frequency of needlesticks was significantly iffected by changes in disposal box design and puncture resistance. Both studies reported reductions in a small subset of cases, those relating to needles protruding from disposable boxes, and those occurring while introducing

\footnotetext{
From the Departments of Neurosurgery and Internal Medicine, University of Virginia School of Medicine, Charlottesville, Virginia.

Address reprint requests to Janine Jagger, MPH, Box 180, Medical Center, University of Virginia, Charlottesville, VA 22908.
}

needles into the boxes. Conversely, neither study reported significant reductions in needlesticks related to other causes, which constituted the vast majority of cases. Despite the failure to reduce the overall rate of needlesticks, the change in disposal systems represents a documented success; one that Ribner and colleagues take one step further by presenting a compelling cost-benefit analysis in support of the continued use of the new system.

It stands to reason that the effects of an innovation would be specific to the new features introduced, rather than generalized to the problem as a whole. The lack of overall impact suggests that the investigators have successfully addressed one small piece in a complex jigsaw of causal factors. Achieving a sizable impact will require an understanding of the many causal factors at work, and will no doubt demand a multifactorial approach to intervention.

The literature on needlestick injuries, which reflects current beliefs about causation, focuses primarily on three factors: 1) the danger of recapping used needles; 2) needle disposal factors, usually related to disposal box design and placement; and 3) the compliance of health care workers with safety recommendations. ${ }^{1-4}$ The range of prevention options inferred by these areas of focus is decidedly limited. Notably lacking in the needlestick literature is a comprehensive inventory of the types and designs of needled instruments that stick health care workers. The opportunity for safety innovations in the designs of needled and sharp devices has yet to be actively addressed.

The National Academy of Science's Committee on Trauma Research reports, "The most successful injuryprevention approaches have involved improved product designs and changes in the man-made environment ..." In order to improve the designs of needled instruments, it is also necessary to determine how they are normally handled in clinical settings and the various circumstances leading to unintentional needlestick injury 
after use. Unfortunately, current data do not provide sufficient detail to lead to improved designs for the wide variety of items causing injury.

This approach is in stark contrast to that of Krasinski and his colleagues, who believe that individual behavior is the most important determinant of needlestick injuries. Statements that "simple control measures are not followed [by employees who engage] in potentially dangerous behavior" boldly suggest that the employee is a perpetrator acting with disregard. Do employees cause needlesticks by their dangerous behavior, or are they victims of inherently dangerous devices which they are required to handle under difficult circumstances? It is revealing that Krasinski and colleagues relied on the notes entered into the Employee Health Service log and Hospital Accident Reports for information on the causes of the injuries. An answer to the question of why an employee would recap a used needle despite recommendations against recapping can be obtained by asking the employee. Ribner and his colleagues interviewed the subjects in their study and the comments are telling: "Those injured during recapping consistently stressed concern over carrying an unsheathed needle back to the central nursing station." A similar dilemma occurs when disassembly of a needled device is required prior to disposal. Rather than acting irresponsibly, many employees attempted to protect themselves from a perceived danger and chose the lesser of two apparent hazards.

It is surprising to note that despite widespread acceptance of the CDC recommendation not to recap, no study has evaluated the benefits or considered the possible risks of refraining from that practice. The CDC recommendation originated from a literature review prior to 1983 in which recapping was frequently cited as a cause of needlestick injury. $2,3,6,7$ Hence, the assumption that avoidance of recapping would result in a proportional decrease in needlesticks. We take issue with the notion that "simple control measures are not followed" by irresponsible employees. Rather, it is likely that currently recommended control measures are too simplistic to be effective in the complex hospital environment.

When viewed as an environmental and product design problem, future prospects for substantially reducing the incidence of needlesticks appear bright since so little attention has been previously paid to the problem. First, it must be determined which items injure health care workers, and the product characteristics associated with the injuries. The best solutions will not require the hands to move toward contaminated needles, nor require the manual disassembly of sharp, contaminated items. Improved devices must perform all of the functions of their hazardous predecessors, and as efficiently. They must be simple, for the purposes of cost containment, and should not require special training or additional equipment to use. ${ }^{8}$ Desirable changes may also require modifications in certain clinical procedures to improve the convenience of handling sharp items.

Replacing the current array of hazardous devices with safer ones will take time, but it will certainly occur if health care providers express their concern for safety in the selection of products for clinical use. Some choices already exist, but many more remain to be developed. It will be the task of the clinical investigators to evaluate the effectiveness of new products in preventing needlestick injuries. There remain important epidemiologic problems to resolve for future evaluation studies. What is the appropriate denominator for determining incidence? Is. it personnel days, patient days, or number of devices used? This question must be resolved before intra- or inter-institutional comparisons can be made with any degree of confidence. To what degree are needlesticks underreported? A significant proportion of needlesticks go unreported by employees, raising the possibility that relatively small shifts in reporting patterns could obscure changes in the true incidence of needlesticks. ${ }^{9}$ There remains much to be done in the methodologic realm.

The magnitude of the needlestick problem and the potential severity of its consequences provide strong impetus for increased scientific inquiry in this area. Progress will require a sustained effort, involving the implementation and evaluation of many strategies for preventing needlestick and sharp object injuries; a process that would undoubtedly benefit from the recognition and support of the relevant funding agencies.

\section{REFERENCES}

1. Jacobson JT, Burke JP, Conti MT: Injuries of hospital employees from needles and sharp objects. Infect Control 1983; 4:100-102.

2. McCormick RD, Maki DG: Epidemiology of needlestick injuries in hospital personnel. Am J Med 1981; 70:928-932.

3. Osterman CA: Relationship of new disposal unit to risk of needle puncture injuries. Hosp Top 1975; 53:12-13.

4. Ruben FL, Norden CW, Rockwell K, et al: Epidemiology of accidental needle puncture wounds in hospital workers. Am J Med Sci 1983; 286:26-30.

5. Committee on Trauma Research: Injury in America: A Continuing Public Health Problem. Washington, DC, National Academy Press, 1985.

6. Seeff LB, Wright EC, Zimmerman HJ, et al: Type B hepatitis after needle-stick exposure: Prevention with hepatitis B immune globulin; Final report of the Veteran's Administration Cooperative Study. Ann Intern Med 1978; 88:285-293.

7. Williams WW: Centers for Disease Control: Guidelines for Infection Control in Hospital Personnel. Infect Control 1983; 4:326-349.

8. Jagger J, Pearson RD, Brand JJ: Avoiding the hazards of sharp instruments. Lancet 1986; 1:1274.

9. Hamory BH: Underreporting of needle stick injuries in a university hospital. A $m$ J Infect Control 1983; 11:174-177. 